\title{
The Novel Coronavirus 2019-nCoV: Epidemiological Situation and Measures Undertaken in Morocco
}

\author{
Abderrahmane Achbani ${ }^{1,2^{*}}$ (D) Hasnaa Sine $^{3}$ (D), Aziz Naciri ${ }^{4}$ (D), Mohamed Amine Baba ${ }^{1}$ (D), \\ Youssef Bouchriti 1,5 , Ahmed Kharbach ${ }^{1,6}$ \\ ${ }^{1}$ High Institute of Nursing Professions and Technical Health, Agadir, MOROCCO \\ ${ }^{2}$ Laboratory of Cell Biology and Molecular Genetics, Department of Biology, Faculty of Sciences, University Ibn Zohr, Agadir, MOROCCO \\ ${ }^{3}$ Medical Biology, Human and Experimental Pathology and Environment. Faculty Of Medicine and pharmacy, MOHAMED V University Of \\ Rabat, 10100, MOROCCO \\ ${ }^{4}$ High Institute of Nursing Professions and Technical Health, Laayoune, MOROCCO \\ ${ }^{5}$ Faculty of Sciences Ibn Zohr Agadir, MOROCCO \\ ${ }^{6}$ Laboratory of Biostatistics, Clinical Research and Epidemiology (LBRCE), Faculty of Medicine and Pharmacy of Rabat, MOHAMED V \\ University of Rabat, MOROCCO \\ * Corresponding author: Abderrahmane Achbani E-mail: abderrahmane.achbani@gmail.com ORCID: 0000-0002-4262-2399 \\ Received: 25 April 2020 Accepted: 28 April 2020
}

\begin{abstract}
The novel coronavirus 2019-nCoV caused by SARS-CoV-2, has provoked a large global and a major public health problem.

Morocco, like other countries worldwide has not escaped this disease which affects the respiratory system. To reduce human-to-human transmission of COVID-19 Large-scale actions have been implemented to control the current epidemic in the Kingdom.

The aims of this study is to focus on the epidemiological situation of nCov-19 in Morocco between 2 Marsh and 09 April 2020 , as well as the governmental measures undertaken to stop the propagation of this pandemic disease.

With the use of therapeutic protocols based on Chloroquine and other antiviral drugs, and all measures of social distancing as a precautionary measure, the Moroccan government has successfully managing this health crisis.
\end{abstract}

Keywords: Coronavirus, 2019-nCoV, pandemic disease, social distancing measures, therapeutic protocol based on Chloroquine, Morocco

\section{INTRODUCTION}

The first report of Coronavirus 2019 (COVID-19) disease was reported in Wuhan, China, on 31 December 2019 (WHO) among a cluster of patients that presented with an unidentified form of viral pneumonia [1]. Consequently, as the situation has evolved very rapidly, on 11 March 2020, the WHO used the term "pandemic" to refer to this disease. Thus 
far more than 1.3 million people have been affected by the disease, and among them, about 80,000 have died [2].

The novel coronavirus 2019-nCoV caused by SARS-CoV-2, has provoked a large global epidemic and a major public health problem [3]. This new viral disease is the third coronavirus epidemic in the 21st century, after Severe Acute Respiratory Syndrome (SARS) and Middle East Respiratory Syndrome (MERS) [4]. Also, the large droplet transmission and contact transmission are the two main routes of transmission [5].

Besides, the clinical symptoms of 2019-nCoV are similar with those for SARS-CoV and MERS-CoV. The majority of patients develop a fever, a dry cough, and dyspnea [6]. These symptoms occur within 2 to 14 days after infection. Moreover, preliminary data have found that elderly people and persons with special health conditions or a weaker immune system are more vulnerable to be infected with a serious disease $[7,8]$.

Regarding the radiological aspect, the hallmarks of COVID19 infection on imaging were bilateral and peripheral ground-glass and consolidating pulmonary opacities. With a longer time after the onset of symptoms, CT findings were more frequent, including consolidation, bilateral and peripheral disease, greater total lung involvement, linear opacities, "crazy-paving" pattern and the "reverse halo" sign [9].

Many countries in Africa are reinforcing their preparedness for the detection and management of imported nCov-19. According to a modelling study, the kingdom of Morocco was at a moderate risk country in the African continent. For this reason, resources, intensified surveillance and capacity building should be considered urgent priorities [10].

From this perspective, the Moroccan government has responded seriously to the coronavirus pandemic since the outbreak of the disease and has put in place a battery of precautionary measures to stop the spread of the virus after the emergence of the first case of COVID-19 on March 2, 2020 [06:00 PM GMT], confirmed at the laboratory of the Pasteur-Morocco [11].

In dealing with the CoV 2019 pandemic, the Moroccan government, in perfect coordination with the World Health Organization, has taken a series of serious measures. First, the tightening of control at airports, ports and at the various land entry points. In addition, from 13 through 16 March 2020, many other mandatory measures restricting the mobility of the population, including cancelling mass assemblies, the closure of schools, and various arrangements and flexibilities for work at home, have also been introduced with an aim to limit people's contact.

The World Health Organization (WHO) declared a Global Health Emergency in the end of January 2020 [12]. With 63 cases and two recoveries, the Moroccan authorities declared a state of health emergency in their country on 19 March 2020, and have adopted a set with strict measures to avoid spreading the new coronavirus.

The protocol for detection for patients suspected of being infected, the technical reference for the diagnosis of Covid19, according to protocols recommended by the World Health Organization, is the Polymerase Chain Reaction (PCR) to detect SARS CoV-2 positive nucleic acid in nasopharyngeal or oropharyngeal swabs, or bronchoalveolar lavage or tracheal aspiration [13].

For this purpose, first case tested positive for COVID-19 in Morocco was reported 38 days ago on March 02, 2020 [06:00 PM GMT]. Whereas the first case of local transmission was recorded on 13 March 2020 [06:00 PM GMT]. The number of patients reported to have contracted COVID19 was 28 within two-weeks of the date of reporting the first case of the pandemic in Morocco. 9 April 2020 [06:00 PM GMT], there have been 1374 cases of coronavirus disease 2019 (COVID-19), with 97 deaths. While recoveries are registering 12 new cases with 109 recoveries to date. The average age of patients is 48 years, ranging from 2 months to 96 years old, with the majority (53\%) being male. Ninety percent of the cases registered to date are Aboriginal cases.

On March 24, 2020, so that more infected people can be identified early, the Direction of Epidemiology and Disease Control of the Moroccan Ministry of Health updated the definition of a SARS-coV-2 case. Moreover, the both peaks on 23 and 28 March are attributed to the active screening conducted in two clusters of cases, one among the people who participated in an organized trip to Egypt and a second in people who were part in a family ceremony. Thus, screening of health care workers was organized and coincided with the second peak of the outbreak [14].

Cases reported in Morocco increased to $(1,374)$ cases, which is comparable to other countries such as South Africa $(1,934)$, Egypt $(1,699)$ and Algeria $(1,666)$. In this sense, cases registered to date remain lower than those registered in other countries such as Spain $(152,446)$, Italy $(143,626)$, Germany $(113,525)$, France $(86,334)$ and the United Kingdom $(65,077)[15]$.

The measures taken by the Moroccan government, Social distancing, and partial health containment, have probably 


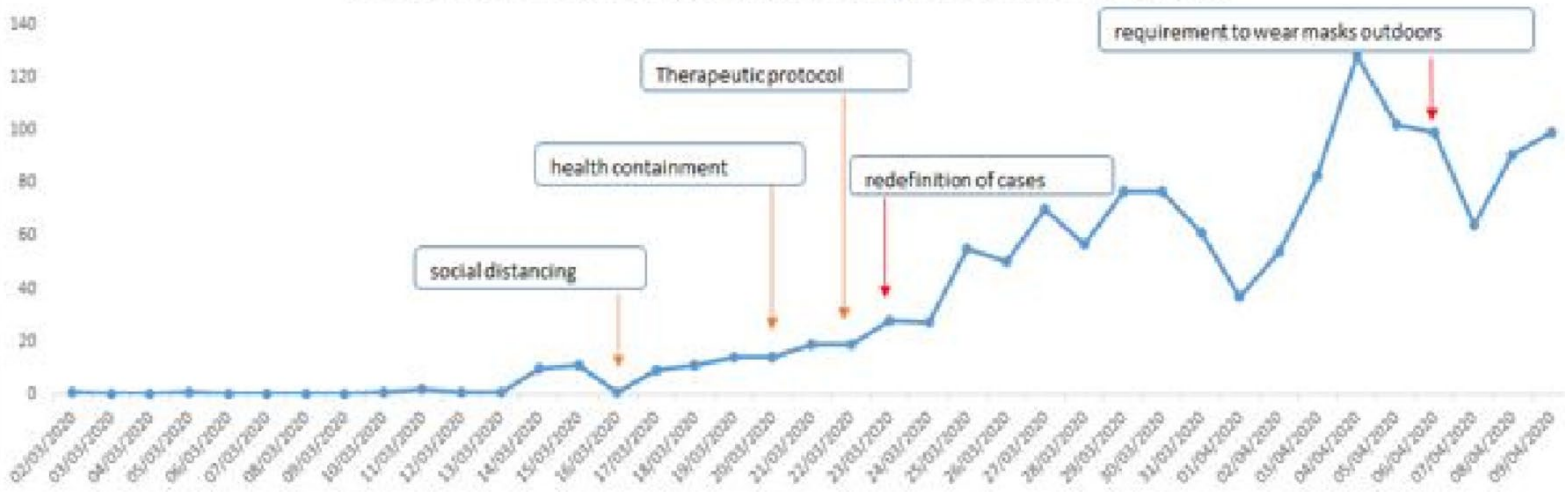

Figure 1. Evolution over time of new cases of COVID-19, Morocco, March 2 to April 9, 2020

Evolution over time of the incidence per million people of COVID-19, Morocco, 2 March - 9 April 2020.

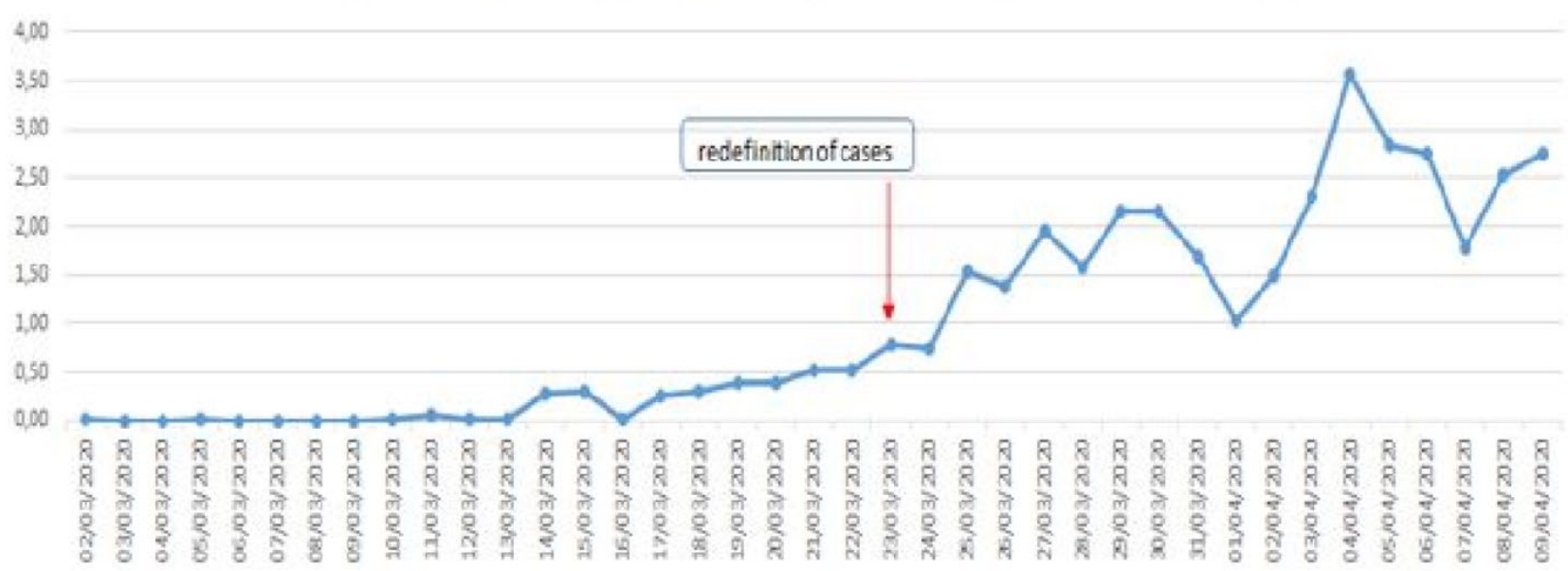

Figure 2. Evolution over time of the incidence per million people of COVID-19, Morocco, March 2 to April 9.2020

contributed to the relative deceleration in the propagation of this pandemic from 29 March 2020. The Health containment means sanitary confinement at home, implemented in response to the spread of the new coronavirus. It is a period during which infectious diseases can be detected and an accurate assessment of the general health status of the population can be made. It also prevents the spread of the virus among the population.

These measures are necessary because limiting social contact of affected people was crucial for the control of COVID-19, as asymptomatic or mildly symptomatic patients can spread the virus [16]. Subsequently, the decision to update the case definition of infection has shown an increase in new positive cases after the onset of the disease in people with no notion of travel (Figure 1) $[17,18]$ and (Figure 2) [17]. This update has taken into consideration the evolution of the national epidemiological situation of COVID-19, marked by the emergence of the disease in some people with no notion of travel.

At the moment, the northern regions seem to be the most affected. These increased incidence rates in the said regions could be explained by the high population density in this

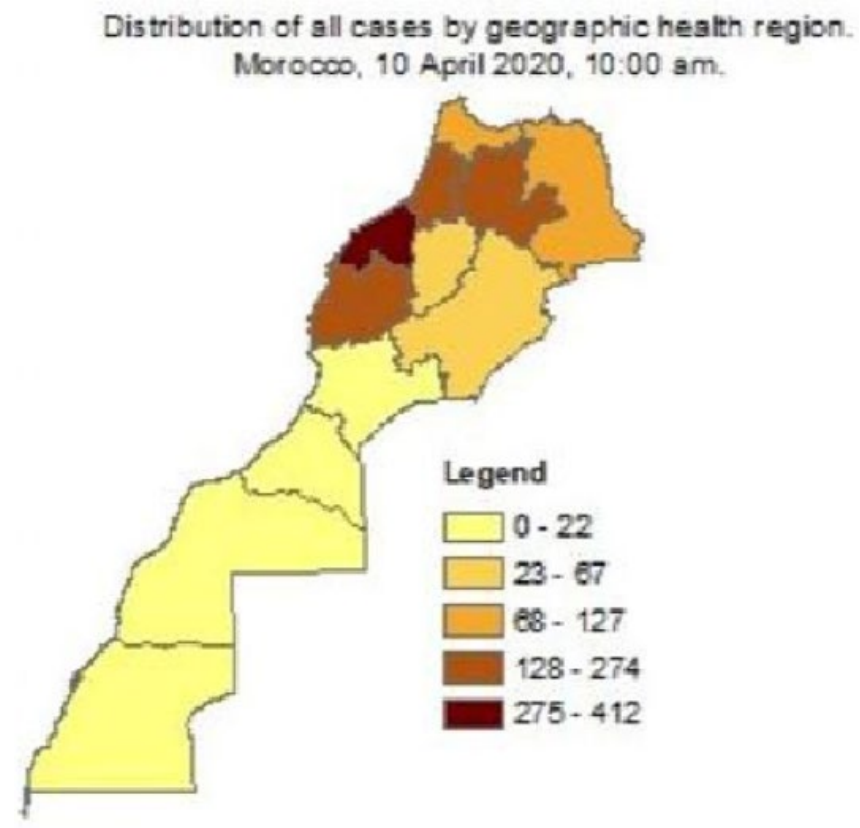

Figure 3. Distribution of cases reported by geographical health region in Morocco, April 10. 2020, 10 h00 GMT

territory of the Kingdom of Morocco. Nevertheless, intensified testing in the southern areas may detect probable cases in the next few days (Figure 3). 


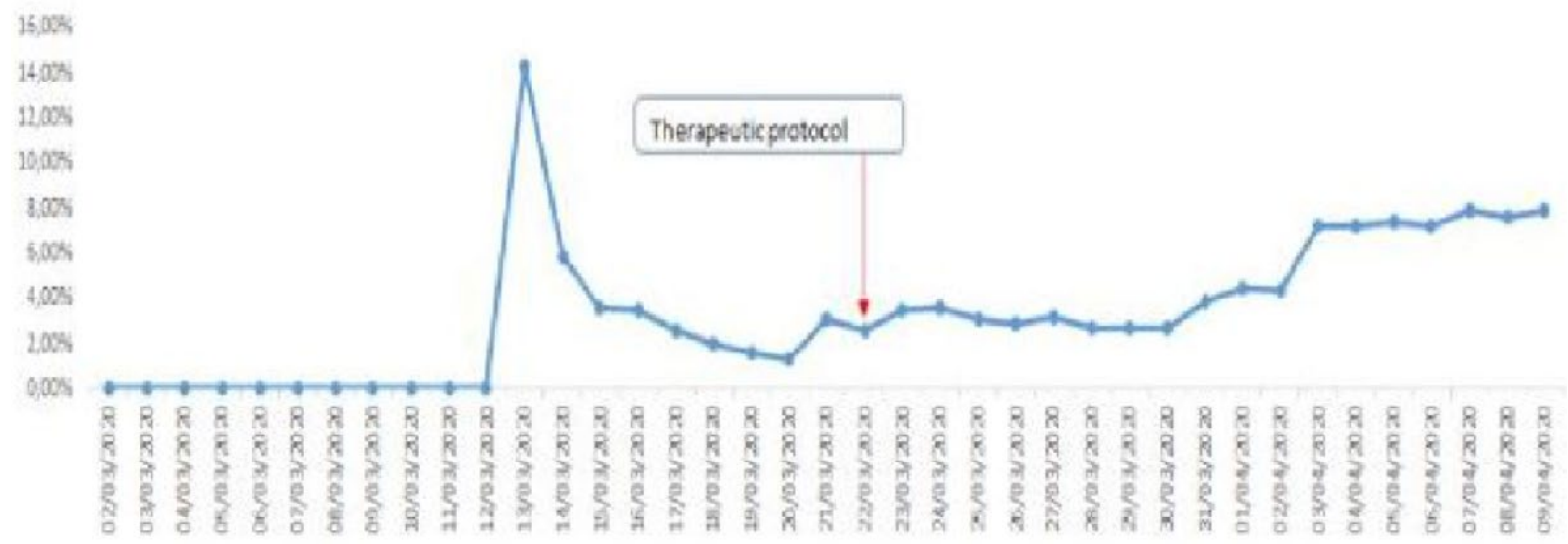

Figure 4. Evolution over time of the recovery rates of COVID-19, Morocco, March 2 to April 9.2020

Evolution over time of the proportion of not yet recovered COVID-19 cases, Morocco, 2 March - 9 April 2020

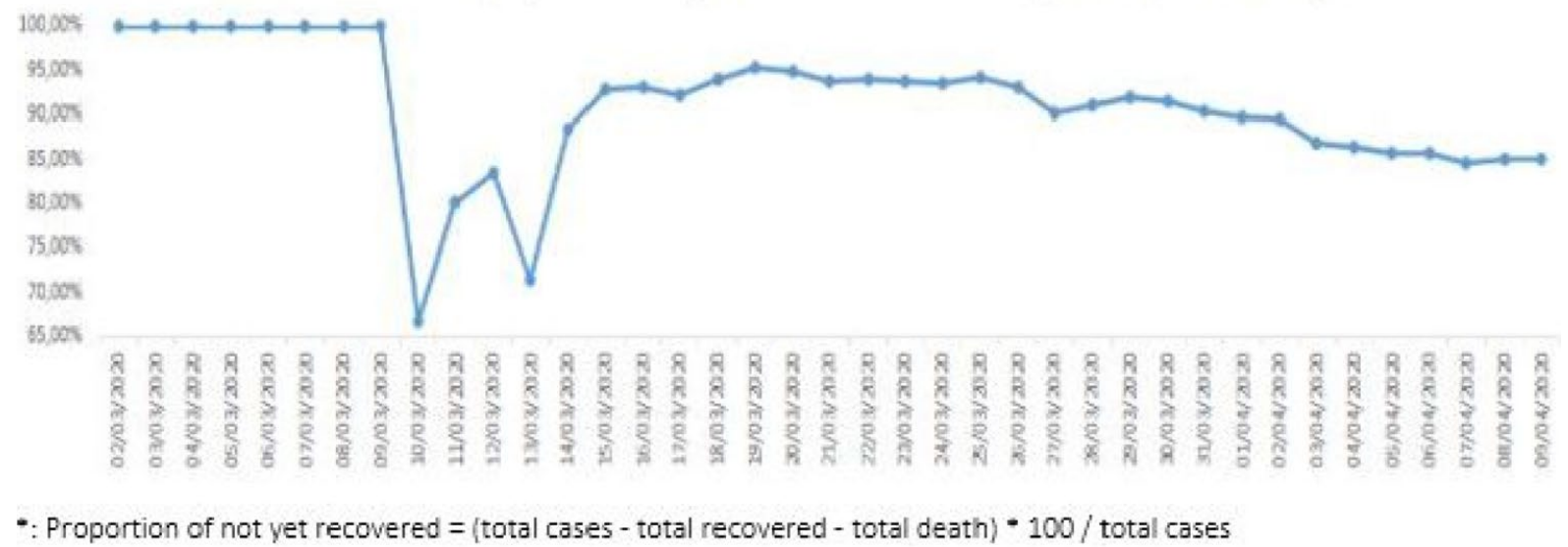

Figure 5. Evolution over time of the proportion of not yet recovered COVID-19, Morocco, March 2 to April 9.2020

The measures have included restrictions of movements and deployment of the security Forces in order to assure respect for these measures. On the basis of recommendations made by the Scientific and technical consultative committee of the national Program for the prevention and Control of influenza and other acute Respiratory Infections, the Ministry of Health has adopted the therapeutic protocol based on Chloroquine and hydroxychloroquine from March 22, 2020. Furthermore, from 6 April 2020, face masks are mandatory in public.

Despite the rigorous process for reporting the recovery of cases, a remarkable evolution of the proportions of recovered cases was observed some ten days after adopting the therapeutic protocol based on the combined prescription of Chloroquine and hydroxychloroquine sulfate in combination with azithromycin and the combination lopinavir/Ritonavir [19]. In addition, as of 9 April, the percentage of cases not yet recovered is estimated at $85 \%$. (Figure 4) [17,18] and (Figure 5) [17].

For mortality in our context, it remains lower than that recorded in other similar countries (Algeria, with 235), and higher in comparison with other countries (Egypt with 103/Tunisia (24)/Burkina Faso (24)) [20]. The provisional apparent mortality rate for COVID-19 is $7.1 \%$ in Morocco, while it seems much lower compared to other countries (Algeria 14.1\%) [18].

The deaths of the confirmed cases of Covid-19 are particularly complicated to analyze. In that sense, several possible scenarios can be considered to interpret of nCov-19 deaths. In the same order of ideas, the lethality rate between 23 March (date of redefinition of cases) and 9 April is between $2.67 \%$ and $7.60 \%$, it suggests that cases are being treated late or detected late. Indeed, as of 9 April, more than $75 \%$ of the cases presented to the health services in a critical situation [14]. Moreover, older people and people with comorbidities (such as asthma, diabetes, heart disease) appear to be more vulnerable to becoming severely ill with the virus [21] (Figure 6) [17,18] and (Figure 7) [17,18].

The relatively large genome of CoVs increases the likelihood of adaptive mutations. These characteristics are probably the cause of the alarming propensity of CoVs to proliferate in the host species [22]. For this reason, health containment, 


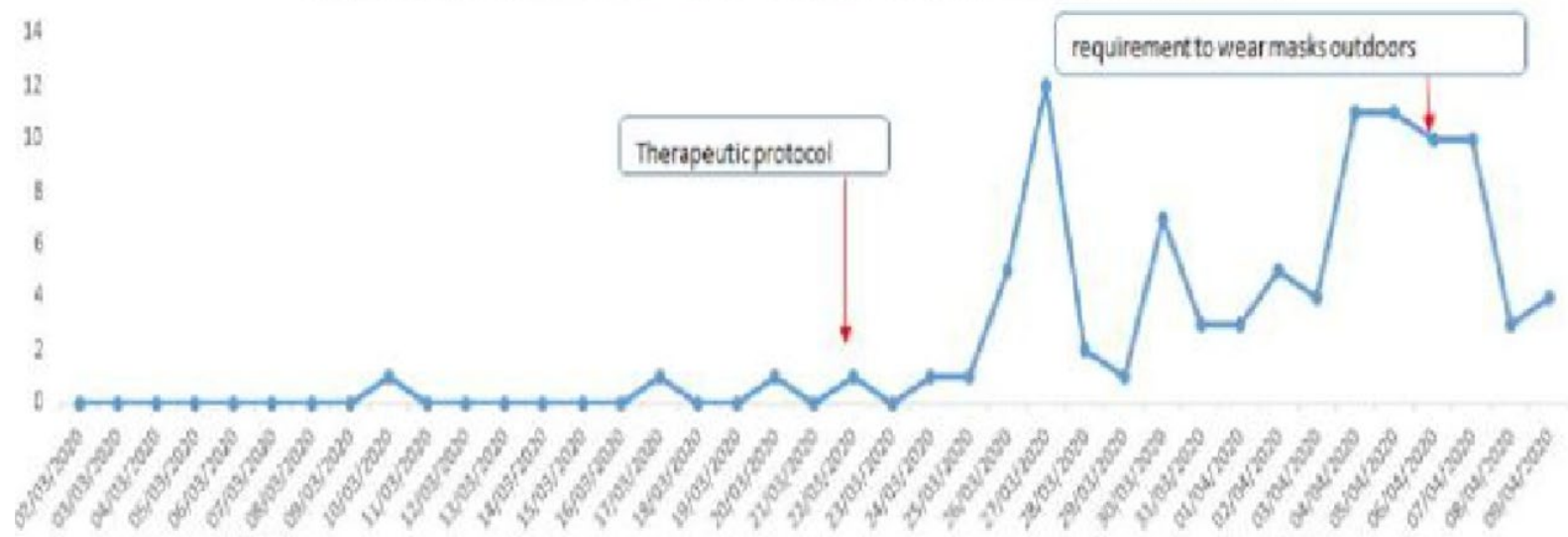

Figure 6. Evolution over time of the new deaths of COVID-19,Morocco,March 2 to April 9.2020

\section{Evalution over time of lethality rate of COVID-19, Morocco, 2 March - 9 April 2020}

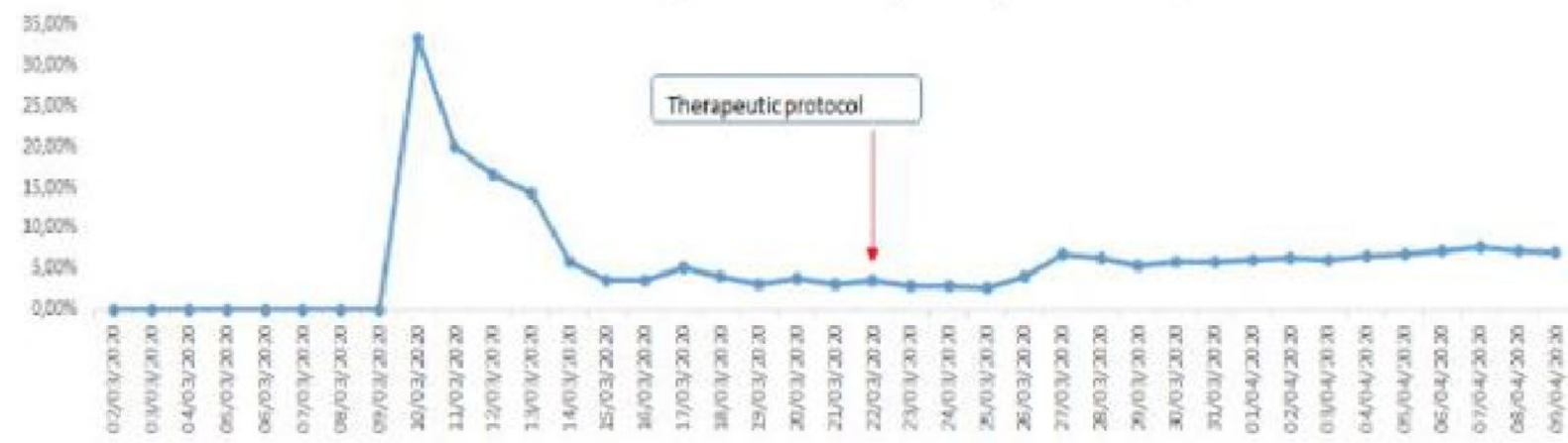

Figure 7. Evolution over time of the lethality rate of COVID-19, Morocco, March 2 to April 9.2020

apart from its social and economic impact, represents a major strategy to decrease the propagation of virus and to reduce drastically influx of patients into health care establishments. The lessons learned will be essential to effectively address similar challenges that may arise in the future. Therefore, the prophylaxis Vaccination is in high demand for the future prevention of new emerging coronaviruses related epidemics or pandemics [23].

\section{DECLARATION OF CONFLICT OF INTEREST}

The authors received no financial support for the research and/or authorship of this article. There is no conflict of interest.

\section{REFERENCES}

1. Zhu N, Zhang D, Wang W, Li X, Yang B, Song J, et al. A novel coronavirus from patients with pneumonia in China, 2019. 2020.

2. World Health Organization. WHO Director-General's opening remarks at the Mission briefing on COVID-19. 2020. Available at: https://bit.ly/2xl10cL (Accessed: 10 April 2020).
3. Lai C-C, Shih T-P, Ko W-C, Tang H-J, Hsueh P-RJljoaa. Severe acute respiratory syndrome coronavirus 2 (SARSCoV-2) and corona virus disease-2019 (COVID-19): the epidemic and the challenges. 2020:105924. (doi: 10.1016/j.ijantimicag.2020.105924).

4. Li J-Y, You Z, Wang Q, Zhou Z-J, Qiu Y, Luo R, et al. The epidemic of 2019-novel-coronavirus (2019-nCoV) pneumonia and insights for emerging infectious diseases in the future. 2020;22(2):80-5. (doi: 10.1016/j.micinf.2020.02.002).

5. Li Q, Guan X, Wu P, Wang X, Zhou L, Tong Y, et al. Early transmission dynamics in Wuhan, China, of novel coronavirus-infected pneumonia. 2020.

6. Huang C, Wang Y, Li X, Ren L, Zhao J, Hu Y, et al. Clinical features of patients infected with 2019 novel coronavirus in Wuhan, China. 2020;395(10223):497-506. (doi: 10.1016/S0140-6736(20)30183-5).

7. Jernigan DBJMM, report mw. Update: public health response to the coronavirus disease 2019 outbreakUnited States, February 24, 2020. 2020;69. (doi: 10.15585/mmwr.mm6908e1). 
8. Eghbali M, Negarandeh R, Froutan RJNPT. COVID-19 epidemic: Hospital-level response. 2020;7(2):81-3. (doi: 10.18502/npt.v7i2.2728).

9. Bernheim A, Mei X, Huang M, Yang Y, Fayad ZA, Zhang N, et al. Chest CT findings in coronavirus disease-19 (COVID19): relationship to duration of infection. 2020:200463. (doi: 10.1148/radiol.2020200463).

10. Gilbert M, Pullano G, Pinotti F, Valdano E, Poletto C, Boëlle $P-Y$, et al. Preparedness and vulnerability of African countries against importations of COVID-19: a modelling study. 2020;395(10227):871-7. (doi: 10.1016/S0140-6736(20)30411-6).

11. Ait Addi R, Benksim A, Amine M, Cherkaoui M. COVID-19 Outbreak and Perspective in Morocco. 2020. Electron J Gen Med. 2020;17(4):em204. (doi: 10.29333/ejgm/7857).

12. Regulations. SotmotlH. the meeting of Emergency Committee regarding the outbreak of novel coronavirus (2019-nCoV). Available at: https://bit.ly/2Rwodiw (Accessed: 10 April 2020).

13. Hadaya J, Schumm M, Livingston EHJJ. Testing Individuals for Coronavirus Disease 2019 (COVID-19). 2020. (doi: 10.1001/jama.2020.5388).

14. Ministry of Health. Epidemic of COVID-19 in Morocco. 2020. Available at: http://www.covidmaroc.ma/Pages/ SituationCovidAR.aspx (Accessed: April 9, 2020).

15. Control. ECfDPa. Situation update worldwide, as of 10 April 2020. Available at: https://www.ecdc.europa.eu/ en/geographical-distribution-2019-ncov-ca (Accessed: 10 April 2020)
16. Guan W-j, Ni Z-y, Hu Y, Liang W-h, Ou C-q, He J-x, et al. Clinical characteristics of 2019 novel coronavirus infection in China. 2020. (doi: 10.1101/2020.02.06.20020974).

17. Oh YS, Kim JS, Yoo SW, Hwang EJ, Lyoo CH, Lee KSJEjon. Gender difference in the effect of uric acid on striatal dopamine in early Parkinson's disease. 2019. (doi: 10.1111/ene.14070).

18. Johns Hopkins University. Coronavirus COVID-19 Global Cases by the Center for Systems Science and Engineering (CSSE) at Johns Hopkins University (JHU). 2020. Available at: https://coronavirus.jhu.edu/map.html (Accessed: 10 April 2020).

19. health. Mo. Scientific and technical consultative committee of the national Program for the prevention and Control of influenza and other acute Respiratory Infections. 2020.

20. Control. Situation ECfDPa. Situation update worldwide, as of 11 April 2020. Available at: https://bit.ly/2ySjnpW (Accessed: 10 April 2020).

21. Vincent J-L, Taccone FSJTLRM. Understanding pathways to death in patients with COVID-19. 2020. (doi: 10.1016/S2213-2600(20)30165-X).

22. Sun J, He W-T, Wang L, Lai A, Ji X, Zhai X, et al. COVID-19: epidemiology, evolution, and cross-disciplinary perspectives. 2020. (doi: 10.1016/j.molmed.2020.02.008).

23. Wang Y, Wang Y, Chen Y, Qin QJJomv. Unique epidemiological and clinical features of the emerging 2019 novel coronavirus pneumonia (COVID-19) implicate special control measures. 2020. (doi: 10.1002/jmv.25748). 